\title{
Réponse des auteurs
}

\section{Bertrand Baumann ${ }^{1}{ }^{*}$, Caroline Ridoux ${ }^{2}$, Véronique Lindner ${ }^{3}$, Pierre Saez ${ }^{2}$}

1 Service d'Odontologie, Centre hospitalier Emile Muller, Mulhouse, France

2 Service d'ORL, Centre hospitalier Emile Muller, Mulhouse, France

3 Service de Pathologie, Centre hospitalier Emile Muller, Mulhouse, France

Nous allons tenter de répondre le plus précisément possible à l'ensemble des questions soulevées dans cette lettre à la rédaction.

Le risque de fracture mandibulaire évoqué lors de la description du cas clinique semblait bien réel, les corticales étant radiologiquement préservées mais très amincies. Des complications pouvaient donc survenir à plusieurs étapes :

- lors d'un accident domestique ;

- lors de l'anesthésie générale, au moment de l'intubation, lors du passage de la sonde d'intubation, l'ouverture buccale forcée obtenue avec le laryngoscope s'accompagne d'importantes sollicitations sur la mandibule ;

- lors du geste opératoire, l'ouverture buccale est maintenue grâce à un ouvre-bouche placé dans le secteur incisif, ce qui entraîne d'importantes sollicitations sur les angles mandibulaires ;

- lors de l'exérèse de la lésion osseuse, après examen extemporané, le diagnostic de kyste odontogène a été posé. Comme il a été précisé dans le protocole opératoire (et comme il est recommandé dans la littérature pour le traitement des kératokystes $[3,5,8])$, un curetage chirurgical a été réalisé. Il en a résulté une diminution de l'épaisseur des corticales bordant la lésion, donc une fragilisation de la mandibule.

Pour l'ensemble de ces raisons, le risque de fracture mandibulaire ne pouvait être négligé et il a été rappelé au patient tout au long de sa prise en charge.

Pour l'examen extemporané demandé, Véronique Lindner apporte les précisions suivantes. Après l'examen extemporané, le diagnostic formulé a été le suivant : "Kyste à revêtement malpighien parakératosique sur le prélèvement examiné. Possible kyste odontogène. Résultat définitif en différé ». L'examen extemporané semblait indiqué pour éliminer un améloblastome ou une lésion tumorale maligne. Les éléments cliniques et radiologiques, même associés aux antécédents du patient, ne permettaient pas d'exclure une lésion améloblastique ou néoplasique. Le résultat fourni constituait un diagnostic d'orientation qui nécessitait d'être complété par l'examen de la totalité de la pièce en technique standard.

Dans ce cas, la formation kystique a effectivement été incluse en totalité et l'examen a révélé « un aspect assez homogène à l'examen histologique. Le même revêtement malpighien sans atypie, reposant sur une lame de tissu conjonctif fibrooedémateux, était observé ; il était surmonté par une discrète parakératose et associé à des lames de kératine exfoliées ». Cet examen en technique standard a ainsi permis de confirmer le diagnostic définitif de kyste odontogène de type kératokyste.

Quant à l'hypoesthésie labio-mentonnière il est vrai que son évolution n'était pas précisée. Après l'intervention, le patient présentait la même hypoesthésie labio-mentionnière qu'en pré- opératoire. Cependant, une régression complète de celle-ci a été observée en 4 semaines. Après 6 mois, la situation clinique était stable et aucun signe de Vincent résiduel n'a été mis en évidence. Cette hypoesthésie labio-mentonière peutêtre expliquée soit par la compression du nerf alvéolaire inférieur soit par un processus inflammatoire induit localement [2] dans le contexte du développement de cette lésion bénigne $[1,4,6,7,9]$.

\section{Conflits d'intérêt : aucun}

\section{Références}

1. Aziz SR, Pulse C, Dourmas MA, Roser SM. Inferior alveolar nerve paresthesia associated with a mandibular dentigerous cyst. J Oral Maxillofac Surg 2002;60:457-9.

2. Baskaran RK, Krishnamoorthy, Smith M. Numb chin syndrome a reflection of systemic malignancy. World J Surg Oncol 2006;4:52.

3. Bradley PF, Fischer AD. The cryosurgery of bone. An expérimental and clinical assessment. Br J Oral Surg 1975;13:111-27.

4. Brannon RB. The odontogenic keratocyst. A clinicopathologic study of 312 cases. Part I. Clinical features. Oral Surg Oral Med Pathol 1976;42:54-72.

5. Le Toux G, Ales RP, Mounier C. Approche chirurgicale des kératokystes odontogènes : à propos de deux cas cliniques. Med Buccale Chir Buccale 2001;7:33-41.

\footnotetext{
*Correspondance : baumannb@ch-mulhouse.fr
} 
6. Lovas JG. Cysts of the jaws: a review. J Can Dent Assoc 1991;57:209-12.

7. Partridge M, Towers JF. The primordial cyst (odontogenic keratocyst): its tumour-like characteristics and behaviour. J Oral Maxillofac Surg 1987;25:271-9.

8. Scharffetter K, Balz-Hermann C, Lagrange W, Kroberg W, Mittermayer C. Proliferation kinetics-study of the growth of keratocysts. Morphofunctionnel exploration of recurrences. J Craniomaxillofac Surg 1989;17:223-6.

9. Seve P, Gachon E, Petiot P, Stankovic K, Charhon A, Broussolle C. Successful treatment with rituximab in a patient with mental nerve neuropathy in primary Sjögren's syndrome. Rheumatol Int 2007;28:175-7. 